\title{
Association between aging-dependent gut microbiome dysbiosis and dry eye severity in C57BL/6 male mouse model: a pilot study
}

Chang Ho Yoon ${ }^{1,2,3}$, Jin Suk Ryu ${ }^{3}$, Jayoon Moon ${ }^{1,2,3}$ and Mee Kum Kim ${ }^{1,2,3^{*}}$

\begin{abstract}
Background: While aging is a potent risk factor of dry eye disease, age-related gut dysbiosis is associated with inflammation and chronic geriatric diseases. Emerging evidence have demonstrated that gut dysbiosis contributes to the pathophysiology or exacerbation of ocular diseases including dry eye disease. However, the relationship between agingrelated changes in gut microbiota and dry eye disease has not been elucidated. In this pilot study, we investigated the association between aging-dependent microbiome changes and dry eye severity in C57BL/6 male mice.

Results: Eight-week-old ( $8 \mathrm{~W}, n=15)$, one-year-old ( $1 \mathrm{Y}, n=10)$, and two-year-old ( $2 \mathrm{Y}, n=8) \mathrm{C} 57 \mathrm{BL} / 6$ male mice were used. Dry eye severity was assessed by corneal staining scores and tear secretion. Bacterial genomic $16 \mathrm{~s}$ rRNA from feces was analyzed. Main outcomes were microbiome compositional differences among the groups and their correlation to dry eye severity. In aged mice (1Y and 2Y), corneal staining increased and tear secretion decreased with statistical significance. Gut microbiome a-diversity was not different among the groups. However, $\beta$-diversity was significantly different among the groups. In univariate analysis, phylum Firmicutes, Proteobacteria, and Cyanobacteria, Firmicutes/Bacteroidetes ratio, and genus Alistipes, Bacteroides, Prevotella, Paraprevotella, and Helicobacter were significantly related to dry eye severity. After adjustment of age, multivariate analysis revealed phylum Proteobacteria, Firmicutes/Bacteroidetes ratio, and genus Lactobacillus, Alistipes, Prevotella, Paraprevotella, and Helicobacter to be significantly associated with dry eye severity.
\end{abstract}

Conclusions: Our pilot study suggests that aging-dependent changes in microbiome composition are related to severity of dry eye signs in C57BL/6 male mice.

Keywords: Dry eye, Aging, Microbiome, Paraprevotella, $16 \mathrm{~S}$ rRNA

\footnotetext{
* Correspondence: kmk9@snu.ac.kr

'Department of Ophthalmology, Seoul National University College of Medicine, 103 Daehak-ro, Jongno-gu, Seoul 03080, Republic of Korea

${ }^{2}$ Department of Ophthalmology, Seoul National University Hospital, 101 Daehak-ro, Jongno-gu, Seoul 03080, Republic of Korea

Full list of author information is available at the end of the article
}

(c) The Author(s). 2021 Open Access This article is licensed under a Creative Commons Attribution 4.0 International License, which permits use, sharing, adaptation, distribution and reproduction in any medium or format, as long as you give appropriate credit to the original author(s) and the source, provide a link to the Creative Commons licence, and indicate if changes were made. The images or other third party material in this article are included in the article's Creative Commons licence, unless indicated otherwise in a credit line to the material. If material is not included in the article's Creative Commons licence and your intended use is not permitted by statutory regulation or exceeds the permitted use, you will need to obtain permission directly from the copyright holder. To view a copy of this licence, visit http://creativecommons.org/licenses/by/4.0/. The Creative Commons Public Domain Dedication waiver (http://creativecommons.org/publicdomain/zero/1.0/) applies to the data made available in this article, unless otherwise stated in a credit line to the data. 


\section{Background}

Dry eye disease is a multifactorial disorder in which the tear film homeostasis is lost, accompanied by several ocular symptoms. Tear film instability and ocular surface inflammation may contribute to the disease [1,2]. Given that the prevalence of dry eye disease increases after middle age, aging is one of the critical risk factors for dry eye disease [3, 4]. As the elderly population increases, socio-economic costs are expected to proportionally increase in the near future [5]. Ocular surface is an exposed mucosa constantly subject to external stimuli. Tear film instability induces hyperosmolar stress in ocular surface epithelium, which subsequently acts as an initial precipitating factor for dry eye disease. Hyperosmolar stress promotes the expression of inflammatory cytokines in the damaged ocular surface epithelium [1]. The inflammatory cytokines may recruit innate immune cells, activate dendritic cells and stimulate T helper 1 (Th1) and 17 (Th17) cells that infiltrate the ocular surface and lacrimal glands $[1,6]$. Regulatory $\mathrm{T}$ cell $\left(\mathrm{T}_{\mathrm{reg}}\right)$ dysfunction is also involved in the progression to chronic dry eye disease [7].

Gut microbes influence the maturation and development of the immune system, interact with the nervous system, and maintain metabolic homeostasis [8-10]. Emerging evidence have implied that gut dysbiosis contributes to the pathophysiology or exacerbation of intestinal diseases as well as systemic diseases including systemic lupus erythematosus and rheumatoid arthritis [11-14]. Moreover, recent studies have demonstrated that alterations in gut microbiota may lead to several ocular diseases including autoimmune uveitis, age related macular degeneration, and dry eye associated with Sjögren's syndrome [15-18]. A recent review also indicates that gut microbiota may interact with the eye [19].

The gut microbiota changes with aging and this agingrelated microbiota alteration is associated with inflammaging and chronic geriatric diseases [20]. Given that the gut dysbiosis is associated with dry eye disease [19], aging-related microbiome dysbiosis may also contribute to aging-related dry eye disease. Therefore, in this pilot study, we aimed to investigate (1) whether aging-related dysbiosis is present in C57BL/6 (B6) male mice and (2) which taxa that altered with aging may be associated with the severity of dry eye. These results will open the door for microbiota modulation in aging-related dry eye disease as one of the treatment options.

\section{Results}

First, we discovered that dry eye developed according to age in $\mathrm{B} 6$ male mice. The mean ( \pm standard deviation (SD)) corneal staining scores (punctate epithelial erosions) of eight-week-old ( $8 \mathrm{~W}$; young), one-year-old (1Y; middle aged), and two-year-old (2Y; elderly) groups were $1.35 \pm$ $1.03,6.25 \pm 1.78$, and $4.81 \pm 1.62$, respectively (Fig. 1a-b).
The scores of the $1 Y$ and $2 Y$ groups were significantly higher than that of the $8 \mathrm{~W}$ group $(p<0.001$ and $p=$ 0.005 , respectively); however, there was no difference between the $1 Y$ and $2 Y$ groups ( $p>0.999$; Kruskal-Wallis test followed by Dunn's post hoc test) (Fig. 1a-b). The amount of tear secretion was not different among the groups; whereas, the correction value according to body weight (BW), which is also clinically relevant, was significantly lower in both $1 \mathrm{Y}$ and $2 \mathrm{Y}$ groups than in the $8 \mathrm{~W}$ group (Fig. 1c; $p=0.003$ and $p=0.044$, respectively; Kruskal-Wallis test followed by Dunn's post hoc test). Supplementary Table 1 shows all measurements of each mouse.

Next, we compared each groups' gut microbiome. Mean $( \pm$ SD) reads per sample was 44,711 ( $\pm 10,442)$ and individual samples ranged from 28,903 to 79,815 reads. The number of operational taxonomic units (OTUs) of each group were not different $(p=0.777$; Kruskal-Wallis test; Fig. 2a). Chao 1 index and Shannon index did not show any significant differences among the groups ( $p=0.207$ and $p=0.395$, respectively; Kruskal-Wallis test; Fig. 2b-c). However, $\beta$-diversity of each groups' gut microbiome analyzed by UniFrac principal coordinates analysis (PCoA) was significantly different among the groups $(p=0.001,8 \mathrm{~W}$ versus (vs.) $1 \mathrm{Y} ; p=0.001$, $8 \mathrm{~W}$ vs. $2 \mathrm{Y}$; and $p=0.009,1 \mathrm{Y}$ vs. $2 \mathrm{Y}$; Permutational multivariate analysis of variance (PERMANOVA)) (Fig. 2d).

Thereafter, we analyzed compositional differences of gut microbiome at the level of phylum, family, and genus of each group (Fig. 3a). At the phylum level (Fig. 3b), the 2Y group exhibited relatively more Firmicutes than the $1 Y$ group $(p=0.037)$, and decreased Bacteroidetes compared to both $8 \mathrm{~W}$ and $1 Y$ groups $(p=0.004$ and $p=0.024$, respectively; Kruskal-Wallis test followed by Dunn's post hoc test). Firmicutes/Bacteroidetes (F/B) ratio was greater in the $2 \mathrm{Y}$ group than the $8 \mathrm{~W}$ and $1 \mathrm{Y}$ groups ( $p=0.004$ and $p=0.024$, respectively; KruskalWallis test followed by Dunn's post hoc test). The $1 Y$ group revealed relatively higher proportion of Proteobacteria (1Y vs. $8 \mathrm{~W}, p=0.049$ ), and lower proportion of Actinobacteria (1Y vs $8 \mathrm{~W}, p=0.002 ; 1 \mathrm{Y}$ vs $2 \mathrm{Y}, p=$ 0.008; Kruskal-Wallis test followed by Dunn's post hoc test). The aged group ( $1 \mathrm{Y}$ and $2 \mathrm{Y}$ ) showed a significantly abundant Cyanobacteria compared to the $8 \mathrm{~W}$ group ( $p=0.002$ and $p<0.001$, respectively). At the family level (Fig. 3c), the aged group had high proportion of Bacteroidaceae and Rikenellaceae, and low proportion of Muribaculaceae compared to the $8 \mathrm{~W}$ group (all $p<0.05$ ). Family Prevotellaceae reduced with aging and the $2 \mathrm{Y}$ group showed a significant reduction compared to the 8 $\mathrm{W}$ group $(p<0.001)$. At the genus level (Fig. 3d), the aged group showed higher proportion of Alistipes and Bacteroides, and lower proportion of Paraprevotella than the $8 \mathrm{~W}$ group (all $p<0.05$ ). The $2 \mathrm{Y}$ group revealed significant reduction in Prevotella compared to both $8 \mathrm{~W}$ 
a

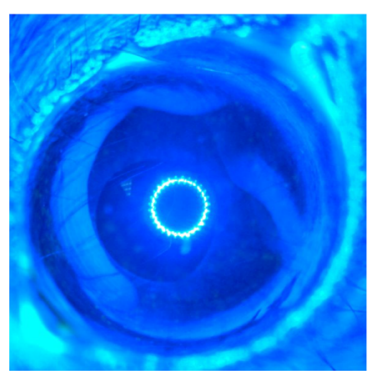

$1 Y$

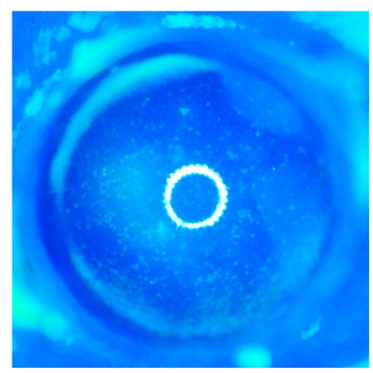

C Tear secretion
$2 Y$

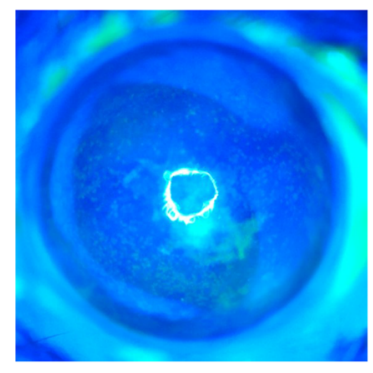

Tear secretion/BW b

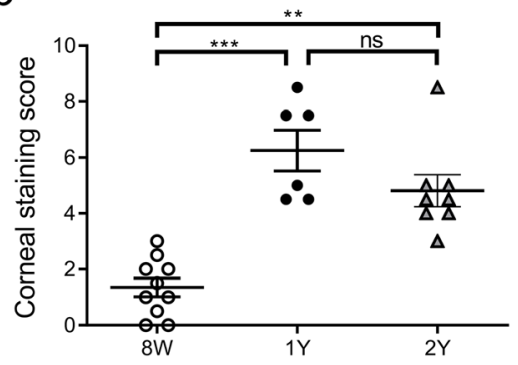

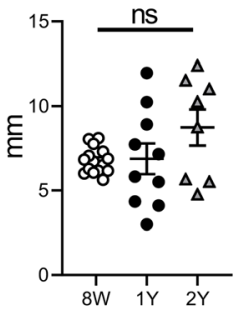



Fig. 1 Corneal staining and tear secretion of the 8 W, $1 Y$, and $2 Y$ groups. a Representative images of corneal fluorescein staining. b The National Eye Institute corneal staining score was significantly higher in one-year-old (1Y) and two-year-old (2Y) mice than in eight-week-old (8 W) mice (all $p<0.01$; Kruskal-Wallis test followed by Dunn's post hoc test; $n=10,6$, and 8 for $8 \mathrm{~W}, 1 Y$, and $2 Y$ mice, respectively). c Tear secretion did not change over time (all $p>0.05$; Kruskal-Wallis test). However, when the level was normalized by body weight (BW), the normalized value was lower in both $1 Y$ and $2 Y$ groups than in the $8 \mathrm{~W}$ group (all $p<0.05$; Kruskal-Wallis test followed by Dunn's post hoc test; $n=15,10$, and 8 for 8 $W, 1 Y$, and $2 Y$ mice, respectively). ns, not significant; ${ }^{*} p<0.05,{ }^{*} p<0.01$, and ${ }^{* *} p<0.001$. Data are presented as means \pm standard error

and 1 Y groups (all $p<0.05$; Kruskal-Wallis test followed by Dunn's post hoc test).

The linear discriminant analysis (LDA) of effect size (LEfSe) analysis was performed to explore the specific taxa that had significant difference among the $8 \mathrm{~W}, 1 \mathrm{Y}$, and $2 \mathrm{Y}$ groups (Fig. 4a), or between the aged (1Y and $2 \mathrm{Y}$ ) and $8 \mathrm{~W}$ young (Fig. $4 \mathrm{~b}$ ) groups. The relative abundance of nine microbial taxa, such as Alistipes (genus), Firmicutes (phylum), Cyanobacteria (phylum), in the $2 \mathrm{Y}$ group was higher compared to both $1 \mathrm{Y}$ and $8 \mathrm{~W}$ groups. Six microbial taxa, such as Bacteroidetes (phylum) and Prevotella (genus), were significantly enriched in $8 \mathrm{~W}$ group. For additional analysis, we combined the data from $1 \mathrm{Y}$ and $2 \mathrm{Y}$ groups into the aged group. Ten microbial taxa in the aged group, such as Alistipes (genus) and Cyanobacteria (phylum), were higher in abundance compared to the $8 \mathrm{~W}$ group, whereas six microbial taxa in the young $8 \mathrm{~W}$ group, such as Paraprevotella (genus), Bacteroidetes (phylum), and Prevotella (genus), were higher in abundance compared to the aged group.

Next, we investigated the relationship between agingdependent changes of gut microbiome and dry eye severity signs using Spearman's rank correlation test. In phylum level (Fig. 5), corneal staining score was positively related with Cyanobacteria $(r=0.584, p=0.003)$.
Tear secretion had negative relation with Proteobacteria $(r=-0.421, p=0.015)$. BW adjusted tear secretion was negatively correlated with Cyanobacteria $(r=-0.494$, $p=0.004$ ). In genus level (Fig. 6), corneal staining score was positively related with Alistipes $(r=0.639, p<0.001)$ and Bacteroides ( $r=0.669, p<0.001)$, and negatively related with Paraprevotella $(r=-0.623, p=0.001)$. Tear secretion had negative relation with Prevotella $(r=-$ 0.439, $p=0.011)$ and Helicobacter $(r=-0.455, p=0.008)$. However, BW adjusted tear secretion was negatively correlated with Alistipes $(r=-0.458, p=0.007)$ and Bacteroides $(r=-0.436, p=0.011)$.

Finally, we performed partial rank correlation analysis to adjust the confounding age factor. In phylum level, only Proteobacteria significantly affected corneal staining score $(r=0.418, p=0.047)$, tear secretion $(r=$ $-0.423, p=0.016)$, and tear secretion with BW adjustment $(r=-0.371, p=0.037)$. In genus level, corneal staining score was positively related to Helicobacter $(r=0.423, p=0.046)$. Both tear secretion with and without adjustment for BW were negatively related to Prevotella $(r=-0.444, p=0.011 ; r=-0.472, p=0.006$; respectively), Paraprevotella ( $r=-0.367, p=0.039 ; r=-0.368, p=0.038$; respectively), and Helicobacter $(r=-0.455, p=0.009$; $r=-0.399, p=0.024$; respectively). 



d

Beta Diversity of Genus

Beta Diversity of Species
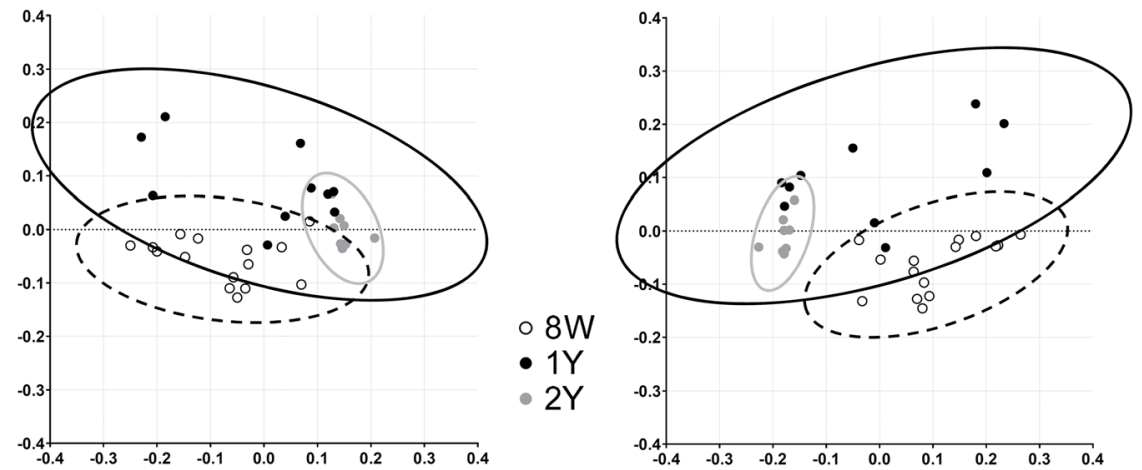

Fig. 2 Alpha and beta diversity analysis of the 8 W, 1 Y, and 2 Y groups. a Observed operational taxonomic unit (OTU) count, (b) Chao 1 index, (c) Shannon diversity index. There were no significant differences among the groups in OTU, Chaol and Shannon index (all $p>0.05$; Kruskal-Wallis test). d $\beta$-diversity of genus and species analyzed by UniFrac principal coordinates analysis revealed to have significant distances between each group ( $p=0.001,8 \mathrm{~W}$ vs. 1Y; $p=$ $0.001,8$ W vs. $2 Y$; and $p=0.009$, $1 Y$ vs. $2 Y$; Permutational multivariate analysis of variance (PERMANOVA)). Bars indicate maximum and minimum values

\section{Discussion}

This study demonstrates that (1) aging-dependent gut dysbiosis is present in B6 male mouse model and (2) dry eye severity is correlated with the gut dysbiosis.

In dry eye disease and Sjögren's syndrome patients, Bacteroidetes, Actinobacteria, and Bifidobacterium were correlated with dry eye severity signs. Sjögren's syndrome patients showed significant gut dysbiosis compared to both healthy and environmental dry eye subjects [17]. In the autoimmune dry eye mouse model, mice fed with a specific probiotics formula resulted in reduced corneal erosion and increased tear volume. A decrease in the inflammatory cytokine, interleukin (IL)$1 \beta$, and increase in the anti-inflammatory cytokine, IL10 , were observed in the probiotics intake group [21]. These seem to be caused by the downregulation of antigen-presenting cells related to gut microbiome changes [21]. Kawashima et al. also reported that dietary supplementation with E. faecium WB2000 mixed with fish oil for 8 weeks improved objective and subjective symptoms with increased tear production in dry eye disease patients [22]. Taken together, dry eye severity signs are associated with gut microbiota dysbiosis, and the consumption of probiotics supplements can alleviate dry eye disease.
In this study, we observed more significant dry eye and related ocular surface damages in aged mice (1Y and $2 \mathrm{Y}$ ) compared to the young group $(8 \mathrm{~W})$ in the $\mathrm{B} 6$ male mouse model. Corneal staining score of $1 Y$ group was comparable to that of $2 \mathrm{Y}$ group. This is in line with previous studies that observed equivalent degree of corneal epithelial defects between 1 and 2 years of age [23], and similar corneal irregularities of 6 to 9 month-old B6 mice when compared to those of 2 year old B6 mice [24]. Aging-related inflammation increases with age, but not in a linear incremental manner [24], and the ocular surface change appears to reach a plateau between 1 and 2 years of age.

We found that the $\alpha$-diversity of the gut microbiome did not change with age. However, $\beta$-diversity and microbiome composition were significantly different according to age, and this difference was clearly observed in the PCoA plot (Fig. 3a). In addition, the distribution in the PCoA plot was very different between $8 \mathrm{~W}$ and $2 \mathrm{Y}$ groups, whereas the $1 \mathrm{Y}$ group lied somewhere in between those groups.

Dysbiosis has been reported in several metabolic disorders such as obesity and hypertension, and appears to increase the relative abundance of F/B ratio [25-27]. High $\mathrm{F} / \mathrm{B}$ ratio is associated with lower butyrate production in 

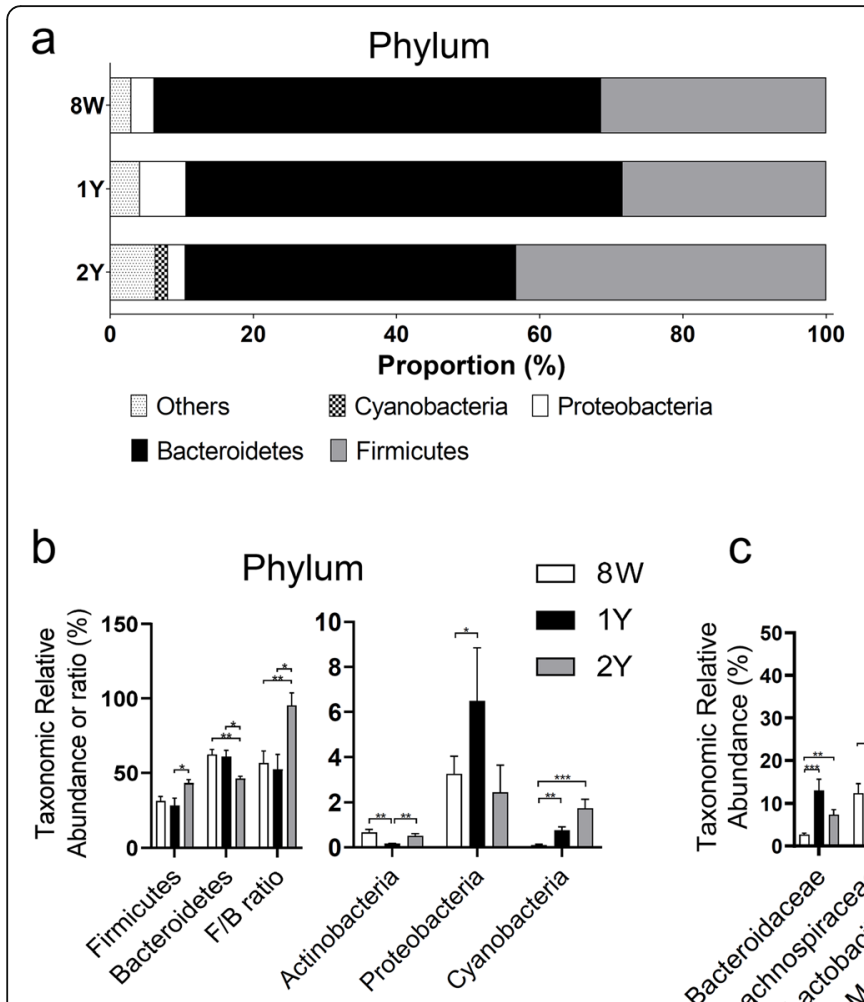


Fig. 3 Taxonomic relative abundance according to phylum, family and genus. a Overall, taxonomic relative abundance in phylum and genus are shown for all groups. $\mathbf{b}$ In phylum, Firmicutes significantly increased (2Y versus (vs.) $1 Y, p=0.037$ ) and Bacteroidetes reduced (2Y vs 8 W, $p=0.004 ; 2 Y$ vs $1 Y, p=0.024)$ in the $2 Y$ group compared to both $8 \mathrm{~W}$ and $1 Y$ groups. Proteobacteria was increased (1Y vs. $8 \mathrm{~W}, p=0.049)$ and Actinobacteria was decreased (1Y vs $8 \mathrm{~W}, p=0.002 ; 1 Y$ vs $2 Y, p=0.008$ ) in the $1 Y$ group. Cyanobacteria was increased in the aged group (1Y and $2 Y$ ) compared to the $8 \mathrm{~W}$ group ( $p=0.002$ and $p<0.001$, respectively). $\mathbf{c}$ In family, Bacteroidaceae and Rikenellaceae were significantly more abundant and Muribaculaceae was significantly reduced in the aged group ( $1 Y$ and $2 Y$ ) compared to the $8 \mathrm{~W}$ group (all $p<0.05$ ). Prevotellaceae was significantly reduced in the $2 \mathrm{Y}$ group than in $8 \mathrm{~W}$ group $(p<0.001)$. $\mathbf{d}$ In genus, the aged group ( $1 Y$ and $2 Y$ ) were increased Alistipes and Bacteroides, and decreased Paraprevotella compared to the $8 \mathrm{~W}$ group (all $p<0.01$ ). The $2 \mathrm{Y}$ group revealed significantly decreased Prevotella than both $8 \mathrm{~W}$ and $1 \mathrm{Y}$ groups (all $p<0.05$ ). ${ }^{*} p<0.05,{ }^{* *} p<0.01$, and ${ }^{* *} p<0.001$ by Kruskal-Wallis test followed by Dunn's post hoc test. Data are presented as means \pm standard error

the gut $[27,28]$. Butyrate, one of short-chain fatty acids (SCFAs), is a preferred energy source for intestinal epithelial cells and has several beneficial clinical effects such as, strengthening the immune system, reducing inflammation, and regulating metabolism [29]. In ocular diseases, high F/ $B$ ratio was observed in experimental autoimmune uveitis and age-related macular degeneration mouse models [30, 31]. In this study, Bacteroidetes was more dominant in the $8 \mathrm{~W}$ group, while Firmicutes was more prevalent in the $2 \mathrm{Y}$ group. In congruence with previous study [32], the F/B ratio increased with age where the $2 \mathrm{Y}$ group showed a significantly higher ratio than the $8 \mathrm{~W}$ and $1 \mathrm{Y}$ groups. These results suggest that gut dysbiosis increases with aging.

Phylum Proteobacteria is a known microbial signature for gut dysbiosis [25]. Gut dysbiosis facilitates anaerobic glycolysis of surface colonocytes, which induces increment of gut epithelial oxygenation, disrupts anaerobiosis in the lumen, and consequently promotes an expansion of facultative anaerobic Proteobacteria [33]. Immunomodulatory cytokine IL-10 deficient mice showed spontaneous development of colitis and had relatively higher proportion of Proteobacteria than wild-type mice [34]. Oral administration of Helicobacter typhlonius (phylum Proteobacteria) triggered tumor necrosis factor (TNF)- $\alpha$ and promoted colitis in $\mathrm{Tbx} 21^{-/-} \operatorname{Rag} 2^{-/-}$ulcerative colitis mice [35]. Increased prevalence of Proteobacteria was observed in metabolic or immune disorders including type II diabetic mellitus, obesity, and systemic lupus erythematosus [36-38]. Among ocular diseases, Proteobacteria was increased in Behçet's disease and age-related macular degeneration [31, 39]. In this study, the abundance of phylum Proteobacteria was increased in the $1 Y$ group. Moreover, correlation analysis revealed that phylum Proteobacteria was positively related to corneal staining and negatively related to tear secretion after age adjustment. Genus Helicobacter also showed a negative correlation with tear secretion regardless of BW adjustment. This genus has been reported to be associated with enteritis and systemic infectious diseases [40]. 


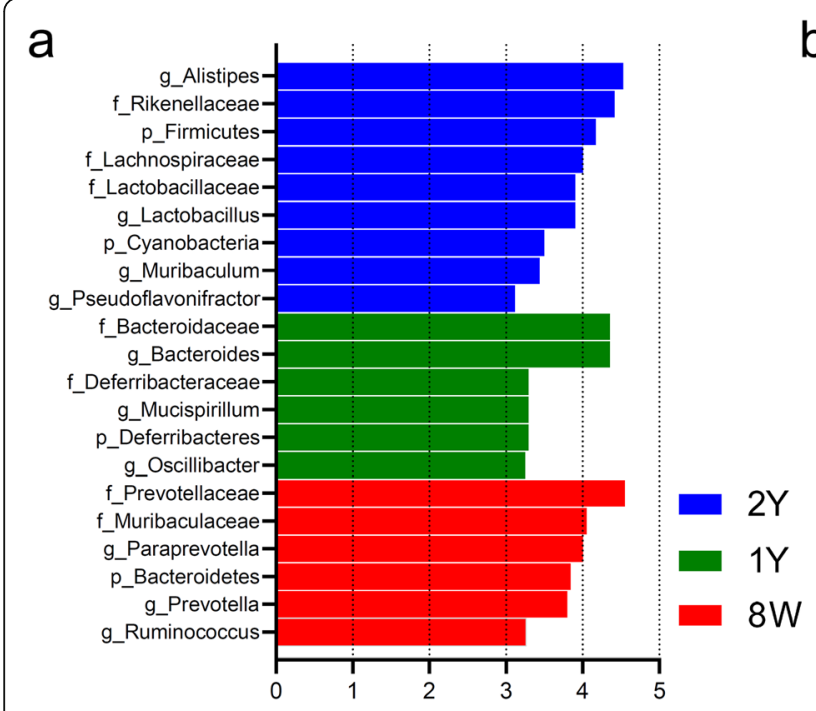

LDA Score (Log 10) b



Fig. 4 Linear discriminant analysis (LDA) effect size (LEfSe) performed on the microbial community relative abundance data. Kruskal-Wallis test produced $p<0.05$ and an LDA score of $>3.0$. a The $2 Y$ group is shown as blue, $1 Y$ as green, and $8 \mathrm{~W}$ as red. $\mathbf{b}$ Combined aged ( $1 \mathrm{Y}+2 \mathrm{Y}$ ) group is shown green, and $8 \mathrm{~W}$ young group as red

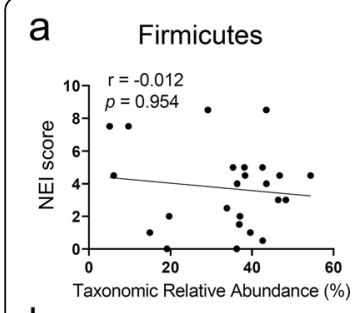

b

Firmicutes

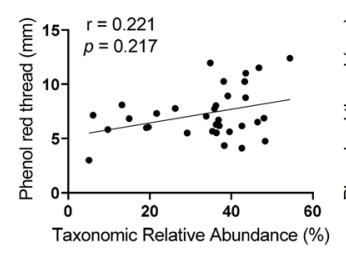

C

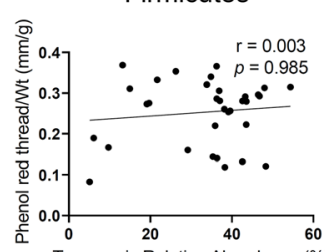

Bacteroidetes

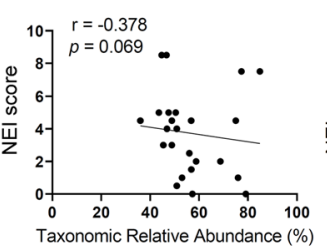

Bacteroidetes

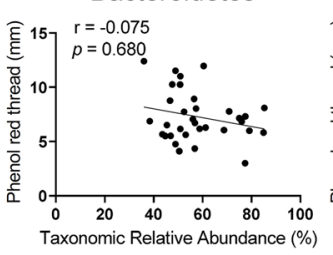

Bacteroidetes

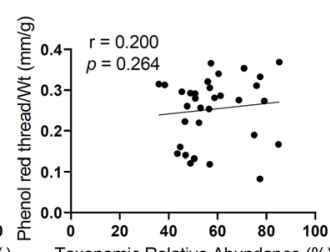

F/B ratio

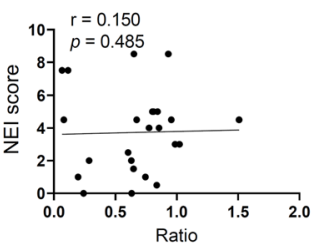

F/B ratio



F/B ratio

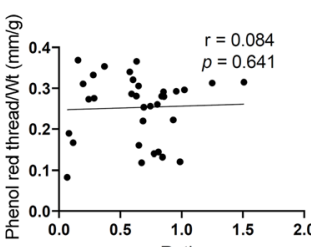

Proteobacteria

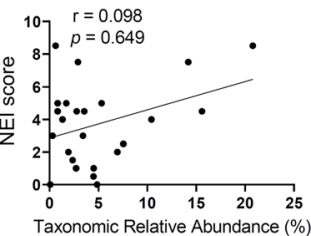

Proteobacteria

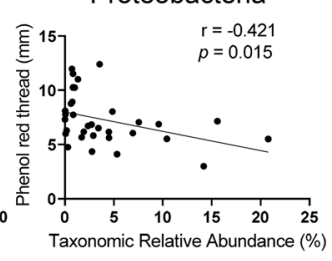

Proteobacteria

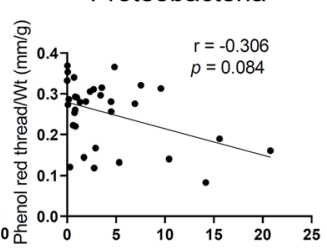

Cyanobacteria

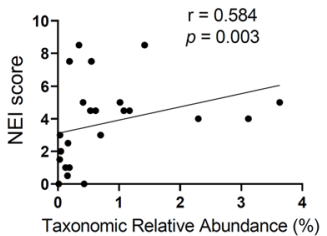

Cyanobacteria



Cyanobacteria

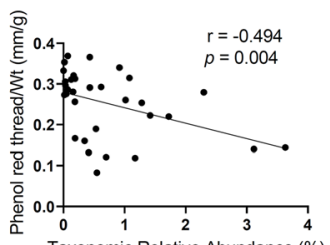

Fig. 5 Spearman's rank correlation analysis between dry eye indices and gut microbiome at the phylum level. a NEl score showed significant positive relation with Cyanobacteria $(r=0.584, p=0.003)$. b Tear secretion was negatively related with Proteobacteria $(r=-0.421, p=0.015)$. c Tear secretion with body weight adjustment showed negative relation with Cyanobacteria $(r=-0.494, p=0.004)$. NEl: National Eye Institute 


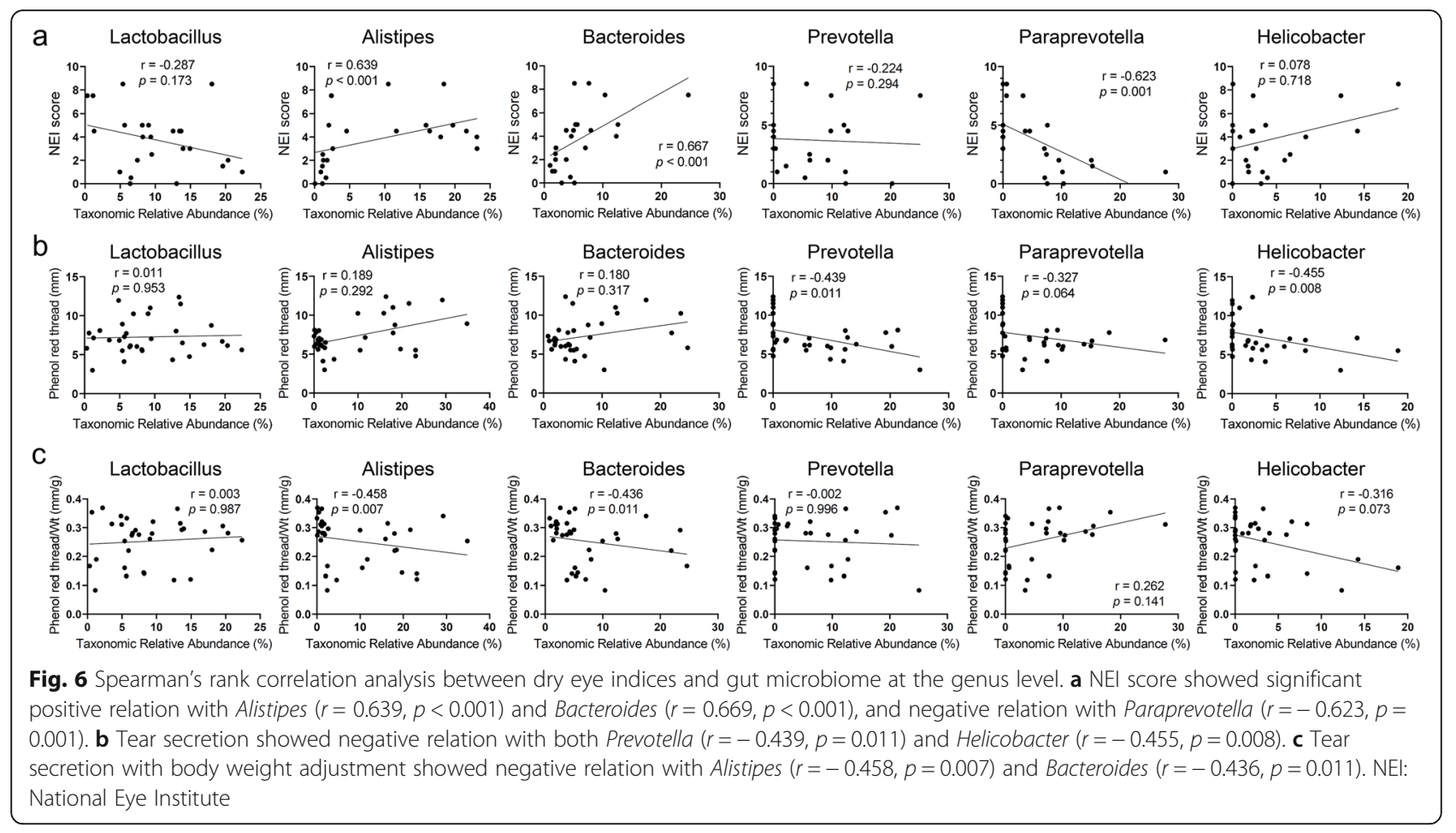

Phylum Cyanobacteria is a bacteria presumably to be involved in the onset of neurodegenerative disease such as Parkinson disease. Cyanobacteria produces neurotoxin b-N-methylamino-L-alanine that may trigger neurodegeneration by promoting mitochondrial dysfunction, protein misfolding, and innate immune responses in genetically susceptible individuals [41]. This phylum was observed to relatively increase in aged mice, and the associated gut dysbiosis was also observed in two different mouse models of progeria where fecal microbiota transplantation from wild-type mice enhanced their lifespan and health [42]. BW adjusted tear secretion was negatively correlated with the Cyanobacteria. Both afferent and efferent neural functions modulating tear production and neurodegeneration induced by Cyanobacteria may play etiological roles [1].

Of note, genus Paraprevotella decreased with aging as reported previously in another study [43]. A decrease in this genus was associated with an increase in corneal staining score. Reduced genus Paraprevotella has been reported in patients with autism spectrum disorder compared to healthy subjects, in low-functioning older adults than in high-functioning older adults, and in sedentary women than in active women [44-46]. In addition, genus Paraprevotella showed negative association with disease staging and motor function of Parkinson disease [47]. This genus produces succinate and acetate as major fermentation products, and only two associated species have been discovered. As described above, the reduction of genus Paraprevotella is related to several diseases but the potential impact on human health is still unknown
$[46,48]$, and it may be connected to the production of acetate, one of the SCFAs.

We also observed that the relative abundance of genus Bacteroides, regarded as an opportunistic pathogen, was significantly increased with age, in agreement with previous studies [49-51]. This genus has been shown to produce virulent factors in the form of polysaccharide that is involved in the destruction of tissues and formation of abscess [52]. The genus Bacteroides was positively correlated with corneal staining score, which is possibly associated with disruptive gut barrier and systemic inflammation.

A suggested pathophysiology of gut dysbiosis associated with dry eye disease may involve modulating effector $\mathrm{T}$ cells directly or via either dendritic cell activation or disturbing gut-derived metabolites [19]. In this study, we found that microbial taxa associated with dysbiosis change with age, and that these microbial taxa were associated with dry eye severity signs. With aging, phylum Proteobacteria and genus Helicobacter (a genus included in phylum Proteobacteria) were positively associated with corneal staining score and negatively associated with tear secretion after age adjustment. The modulation of phylum Proteobacteria may be relevant in the treatment of age-related dry eye disease.

Our study has several limitations. First, a small number of animals were used, and the corneal staining score was not measured in all mice. Therefore, the results may be insufficient to represent each group. However, this study can be used as a pilot study for future research. 
Second, this study was conducted with only male mice. The female sex is one of the potent risk factors for dry eye disease due to hormonal effects [53]. Therefore, it may not be possible to extend the results to female. Third, this was an animal study conducted with mice. However, B6 mice are frequently used in aging research because they develop all the hallmarks of dry eye clinical features including corneal barrier dysfunction, loss of goblet cells, meibomian gland dysfunction, increased dry eye related cytokine expressions, dry eye signs with age, and inflammatory cell infiltrations in lacrimal gland associated with aging [54-56]. Fourth, we did not cohouse mice or use littermates. We included mice of different ages; therefore, we could not use the same littermates. Moreover, mice that have passed the weaning period, especially males, tend to fight until death when co-housed, and so, we could not co-house them [57]. For these reasons, a co-housing or littermate method could not be applied in previous studies comparing gut microbiome of young and old mice [58, 59]. Fifth, relative abundance may not be as accurate as absolute abundance [60]. The overall abundance of bacteria in the gut is likely to also change with age, thereby just showing relative abundance may overlook important key findings. Lastly, we evaluated the association between gut microbiome changes and dry eye severity signs, and we did not reveal any cause-and-effect relationship. Analysis of serum inflammatory cytokines, stool SCFA, or histological changes in the ocular surface with aging will be needed in future studies to strengthen the association of age-related microbiome changes to dry eye severity. Probiotics have been observed to improve dry eye disease severity, suggesting that regulation of gut microbiota might aid future discovery of effective treatment for dry eye disease $[21,22]$.

\section{Conclusions}

Several microbial taxa change in aged mice are related to gut dysbiosis or chronic inflammation, which are possibly associated with the severity of dry eye. In aged mice, phylum Proteobacteria, Cyanobacteria, and genus Bacteroides increased while genus Paraprevotella decreased. These microbial taxa were associated with increased corneal staining score or decreased tear volume. Especially, phylum Proteobacteria was positively associated with corneal staining score. Modulation of gut dysbiosis may be a new therapeutic strategy for age-related dry eye disease.

\section{Method}

The experimental protocol was approved on May 29, 2017 by the Institutional Animal Care and Use Committee of the Seoul National University Biomedical Research Institute (IACUC no. 19-0076-S1A0). Animal experiments were performed in accordance with the ARVO Statement for Use of Animals in Ophthalmic Vision and Research and ARRIVE guidelines. It is known that $\mathrm{B} 6$ mice have increased corneal erosion at 6-9 months of age, and histological changes in the lacrimal glands are evident at 12 months of age [24, 61]. Moreover, mice older than 24 months have a have a higher chance of natural death caused by aging [62]. Therefore, we set 12 and 24 months of age as the time points of the analysis and compared them with 8-week-old mice as controls.

\section{Animals}

A total of 33 B6 male mice were used in this study. Fifteen $8 \mathrm{~W}$, ten $1 \mathrm{Y}$, and eight $2 \mathrm{Y}$ mice were included. All mice were purchased from the same company (KOATECH; Pyeongtaek, Gyeonggi-do, South Korea) at 6-weeks of age, and freely fed a normal diet of laboratory rodent chow (38,057; Cargill Agri Purina, Inc., Seongnam, South Korea). Mice cages were in a specific pathogen-free facility at Seoul National University Hospital Biomedical Research Institute (Seoul, Korea), maintained at $22-24{ }^{\circ} \mathrm{C}$ with $55 \pm 5 \%$ relative humidity, and given food and water ad libitum. After the study, the mice continued to be observed without sacrificing for further study related to aging-related dry eye disease.

\section{Clinical evaluation}

The corneal staining and tear secretion test were performed under anesthesia (using a mixture of xylazine and zoletil at a ratio of 3:1). The corneal staining was evaluated first, and the tear secretion was measured at least $24 \mathrm{~h}$ apart. The corneal staining scores were blindly assigned by a single experienced ophthalmologist ( $\mathrm{CH}$. Y.) according to the National Eye Institute (NEI) grading scale [63]. A drop of $0.25 \%$ fluorescein dye was applied to the conjunctival sac for $30 \mathrm{~s}$, then the ocular surface was gently washed with $1 \mathrm{~mL}$ of normal saline, and corneal staining was evaluated using a microscope (Olympus SZ61; Olympus Corporation, Tokyo, Japan) under cobalt blue illumination. For the tear secretion test, phenol red-impregnated cotton threads (FCI Ophthalmics, Pembroke, MA, USA) were inserted into the lateral canthus of mice for $60 \mathrm{~s}$. The amount of tear secretion was determined by measuring the length of the wet thread in millimeters. The correction value obtained by dividing the amount of tear secretion by the BW of the mouse was also calculated [64]. Corneal staining score was evaluated in ten $8 \mathrm{~W}$, six $1 \mathrm{Y}$, and eight $2 \mathrm{Y}$ mice, and tear secretion test was performed in all mice. The average values of corneal staining scores and tear secretion results from both eyes were used for the analyses. 


\section{Fecal microbiota analysis}

All collected feces were stored at $-80^{\circ} \mathrm{C}$ until they were referred to Chunlab, Inc. (Seoul, Korea) for microbiota analysis as previously described [17]. Total DNA extraction was performed using the FastDNA ${ }^{\bullet}$ SPIN Kit for Soil (MP Biomedicals, USA), in accordance with the manufacturer's instruction. Polymerase chain reaction (PCR) amplification was performed using extracted DNA and bacterial PCR primers 341F (5'-TCGTCG GCAGCGTC-AGATGTGTATAAGAGACAG-CCTA CGGGNGGCWGCAG-3'; underlining sequence indicates the target region primer) and 805R (5'-GTCTCG TGGGCTCGG-AGATGTGTATAAGAGACAG-GACT ACHVGGGTATCTAATCC-3') targeting V3-V4 regions of $16 \mathrm{~S}$ rRNA. The reaction conditions for the first PCR amplification were as follows: $3 \mathrm{~min}$ of initial denaturation at $95^{\circ} \mathrm{C}, 25$ cycles of $30 \mathrm{~s}^{\prime}$ denaturation at $95^{\circ} \mathrm{C}$, $30 \mathrm{~s}$ primer annealing at $55^{\circ} \mathrm{C}, 30 \mathrm{~s}$ elongation at $72^{\circ} \mathrm{C}$, and final extension at $72^{\circ} \mathrm{C}$ for $5 \mathrm{~min}$. The second PCR amplification was performed using i 5 forward primer (5'-AATGATACGGCGACCACCGAGATCTACAC-

XXXXXXXX-TCGTCGGCAGCGTC-3'; X indicates the barcode region) and i7 reverse primer (5'-CAAGCAGA AGACGGCATACGAGAT-XXXXXXXX-GTCTCG

TGGGCTCGG-3') for attaching the Illumina NexTera barcode. The second amplification conditions were the same as those described for the first reaction except only eight amplification cycles were performed. Amplification was confirmed using gel electrophoresis on $1 \%$ agarose gel and visualized under a Gel Doc system (BioRad, Hercules, CA, USA). The product size and quality were assessed on a Bioanalyzer 2100 (Agilent, Palo Alto, CA, USA) using a DNA 7500 chip. Mixed amplicons were pooled and sequencing was performed using an Illumina MiSeq Sequencing system (Illumina, Inc., San Diego, CA, USA) according to the manufacturer's instruction at Chunlab, Inc. (Seoul, Korea). The EzBioCloud database (http://ezbiocloud.net) was used for taxonomic classification after chimera check. To detect chimera on reads that contain lower than $97 \%$ best hit similarity rate, UCHIME and the non-chimeric 16S rRNA database from EzBioCloud were used [17]. The dataset was normalized to the lowest number of read counts (28903 reads per sample) for further analysis.

\section{Statistical analysis}

$\alpha$-diversity analysis expressed with the observed OTUs, Chao1, Shannon, and $\beta$-diversity analysis expressed with generalized UniFrac were carried out using EZBioCloud, a bioinformatics cloud platform of ChunLab Inc. (Seoul, Korea). PCoA was performed to visualize differences in the samples at genus and species level. PERMANOVA was used to evaluate significance in UniFrac PCoA. Compositional abundance differences from each sample were identified by analysis using LEfSe and the KruskalWallis test [65]. Taxa with more than $1 \%$ abundance in at least one group were included in the analysis. Only those taxa that showed a $p$-value $<0.05$ and $\log$ LDA score $\geq 3$ were used as thresholds. For multiple comparisons, the Kruskal-Wallis test followed by Dunn's post hoc test was used. Spearman's rank correlation and partial rank correlations were used to analyze the relationship between measures. Spearman's rank correlation analysis between dry eye indices and clinically important microbials with significant differences among groups was performed. Partial rank correlation analysis was performed to identify independent significant microbials affecting dry eye signs after adjustment of confounding age factor. Statistical analyses were performed using GraphPad Prism software (version 8.2.0; GraphPad Software, La Jolla, CA, USA) and SPSS Statistics 20.0 (IBM Corporation, NY, USA). Differences were considered statistically significant at $p<0.05$.

\section{Abbreviations \\ 1Y: One-year old; 2Y: Two-year-old; 8 W: Eight-week-old; B6: C57BL/6; BW: Body weight; F/B: Firmicutes/Bacteroidetes; IL: Interleukin; LDA: Linear discriminant analysis; LEfSe: Linear discriminant analysis of effect size; NEI: National Eye Institute; OTU: Operational taxonomic unit; PCOA: Principal coordinates analysis; PCR: Polymerase chain reaction; PERM \\ ANOVA: Permutational multivariate analysis of variance; SCFA: Short-chain fatty acid; SD: Standard deviation; Th1: T helper 1 cell; Th17: T helper 17 cell; TNF: Tumor necrosis factor; $T_{\text {reg: }}$ : Regulatory $T$ cell; vs.: Versus}

\section{Supplementary Information}

The online version contains supplementary material available at https://doi. org/10.1186/s12866-021-02173-7.

Additional file 1: Table S1. Corneal staining score, tear secretion, and body weight adjusted tear secretion of each mouse.

\section{Acknowledgements}

Not applicable.

\section{Authors' contributions}

Conceived and designed the experiments: CHY, JSR, MKK; Performed the experiments: CHY, JSR; Analyzed data: CHY, JM, MKK; Write the manuscript: $\mathrm{CHY}, \mathrm{JM}, \mathrm{MKK}$. All authors have read and approved the manuscript.

\section{Funding}

This work was supported by the Cooperative Research Program of Basic Medical Science and Clinical Science from Seoul National University College of Medicine (grant no. 800-20190256) and by the National Research Foundation of Korea (NRF) grant funded by the Korea government (MSIT) (No. 2020R1F1A1072506).

\section{Availability of data and materials}

The datasets used and/or analyzed during the current study are available from the corresponding author on reasonable request.

\section{Declarations}

Ethics approval and consent to participate

The experimental protocol was approved on May 29, 2017 by the Institutional Animal Care and Use Committee of the Seoul National University Biomedical Research Institute (IACUC no. 19-0076-S1A0). 


\section{Consent for publication}

Not applicable.

\section{Competing interests}

The authors declare that they have no competing interests.

\section{Author details}

'Department of Ophthalmology, Seoul National University College of Medicine, 103 Daehak-ro, Jongno-gu, Seoul 03080, Republic of Korea. ${ }^{2}$ Department of Ophthalmology, Seoul National University Hospital, 101 Daehak-ro, Jongno-gu, Seoul 03080, Republic of Korea. ${ }^{3}$ Laboratory of Ocular Regenerative Medicine and Immunology, Biomedical Research Institute, Seoul National University Hospital, 101 Daehak-ro, Jongno-gu, Seoul 03080, Republic of Korea.

Received: 12 December 2020 Accepted: 24 March 2021 Published online: 09 April 2021

\section{References}

1. Bron AJ, de Paiva CS, Chauhan SK, Bonini S, Gabison EE, Jain S, et al. TFOS DEWS II pathophysiology report. Ocul Surf. 2017;15(3):438-510. https://doi. org/10.1016/j.jtos.2017.05.011.

2. Craig JP, Nichols KK, Akpek EK, Caffery B, Dua HS, Joo CK, et al. TFOS DEWS II definition and classification report. Ocul Surf. 2017;15(3):276-83. https:// doi.org/10.1016/j.jtos.2017.05.008.

3. Shanti Y, Shehada R, Bakkar MM, Qaddumi J. Prevalence and associated risk factors of dry eye disease in 16 northern west bank towns in Palestine: a cross-sectional study. BMC Ophthalmol. 2020;20(1):26. https://doi.org/10.11 86/s12886-019-1290-z.

4. Vehof J, Snieder H, Jansonius N, Hammond CJ. Prevalence and risk factors of dry eye in 79,866 participants of the population-based lifelines cohort study in the Netherlands. Ocul Surf. 2020;19:83-93. https://doi.org/10.1016/j. jtos.2020.04.005.

5. Population Division. World Population Prospects 2019. United Nations: Department of Economic and Social Affairs; 2019. https://population.un.org/ wpp/Download/Probabilistic/Population. Accessed 10 Dec 2020

6. Li DQ, Chen Z, Song XJ, Luo L, Pflugfelder SC. Stimulation of matrix metalloproteinases by hyperosmolarity via a JNK pathway in human corneal epithelial cells. Invest Ophthalmol Vis Sci. 2004;45(12):4302-11. https://doi. org/10.1167/iovs.04-0299.

7. Fan NW, Dohlman TH, Foulsham W, McSoley M, Singh RB, Chen Y, et al. The role of Th17 immunity in chronic ocular surface disorders. Ocul Surf. 2020; 19:157-68. https://doi.org/10.1016/j.jtos.2020.05.009.

8. Tremaroli V, Bäckhed F. Functional interactions between the gut microbiota and host metabolism. Nature. 2012;489(7415):242-9. https://doi.org/10.1038/ nature11552.

9. Muller PA, Schneeberger M, Matheis F, Wang P, Kerner Z, llanges A, et al. Microbiota modulate sympathetic neurons via a gut-brain circuit. Nature. 2020:583(7816):441-6. https://doi.org/10.1038/s41586-020-2474-7.

10. Walsh CJ, Guinane CM, O'Toole PW, Cotter PD. Beneficial modulation of the gut microbiota. FEBS Lett. 2014;588(22):4120-30. https://doi.org/10.1016/j. febslet.2014.03.035

11. Barbara G, Scaioli E, Barbaro MR, Biagi E, Laghi L, Cremon C, et al. Gut microbiota, metabolome and immune signatures in patients with uncomplicated diverticular disease. Gut. 2017;66(7):1252-61. https://doi. org/10.1136/gutjnl-2016-312377.

12. Manichanh C, Rigottier-Gois L, Bonnaud E, Gloux K, Pelletier E, Frangeul L, et al. Reduced diversity of faecal microbiota in Crohn's disease revealed by a metagenomic approach. Gut. 2006;55(2):205-11. https://doi.org/10.1136/ gut.2005.073817

13. Luo XM, Edwards MR, Mu Q, Yu Y, Vieson MD, Reilly CM, et al. Gut microbiota in human systemic lupus Erythematosus and a mouse model of lupus. Appl Environ Microbiol. 2018;84(4):e02288-17. https://doi.org/10.112 8/AEM.02288-17.

14. Pernis AB. Th17 cells in rheumatoid arthritis and systemic lupus erythematosus. J Intern Med. 2009;265(6):644-52. https://doi.org/10.1111/j.13 65-2796.2009.02099.x.

15. Horai R, Sen HN, Caspi RR. Commensal microbiota as a potential trigger of autoimmune uveitis. Expert Rev Clin Immunol. 2017;13(4):291-3. https://doi. org/10.1080/1744666X.2017.1288098.
16. Zinkernagel MS, Zysset-Burri DC, Keller I, Berger LE, Leichtle AB, Largiadèr $C R$, et al. Association of the Intestinal Microbiome with the development of Neovascular age-related macular degeneration. Sci Rep. 2017;7(1):40826. https://doi.org/10.1038/srep40826.

17. Moon J, Choi SH, Yoon CH, Kim MK. Gut dysbiosis is prevailing in Sjogren's syndrome and is related to dry eye severity. PLoS One. 2020;15(2):e0229029. https://doi.org/10.1371/journal.pone.0229029.

18. de Paiva CS, Jones DB, Stern ME, Bian F, Moore QL, Corbiere S, et al. Altered mucosal microbiome diversity and disease severity in Sjogren syndrome. Sci Rep. 2016;6(1):23561. https://doi.org/10.1038/srep23561.

19. Moon J, Yoon $\mathrm{CH}$, Choi SH, Kim MK. Can gut microbiota affect dry eye syndrome? Int J Mol Sci. 2020;21(22):8443. https://doi.org/10.3390/ ijms21228443.

20. Nagpal R, Mainali R, Ahmadi S, Wang S, Singh R, Kavanagh K, et al. Gut microbiome and aging: physiological and mechanistic insights. Nutr Healthy Aging. 2018;4(4):267-85. https://doi.org/10.3233/NHA-170030.

21. Choi SH, Oh JW, Ryu JS, Kim HM, Im SH, Kim KP, et al. IRT5 probiotics changes immune modulatory protein expression in the Extraorbital lacrimal glands of an autoimmune dry eye mouse model. Invest Ophthalmol Vis Sci. 2020;61(3):42. https://doi.org/10.1167/iovs.61.3.42.

22. Kawashima M, Nakamura S, Izuta Y, Inoue S, Tsubota K. Dietary supplementation with a combination of Lactoferrin, fish oil, and enterococcus faecium WB2000 for treating dry eye: a rat model and human clinical study. Ocul Surf. 2016;14(2):255-63. https://doi.org/10.1016/j.jtos.201 5.12.005.

23. Matlock HG, Qiu F, Malechka V, Zhou K, Cheng R, Benyajati S, et al. Pathogenic role of PPARa Downregulation in corneal nerve degeneration and impaired corneal sensitivity in diabetes. Diabetes. 2020;69(6):1279-91. https://doi.org/10.2337/db19-0898.

24. McClellan AJ, Volpe EA, Zhang X, Darlington GJ, Li DQ, Pflugfelder SC, et al. Ocular surface disease and dacryoadenitis in aging C57BL/6 mice. Am J Pathol. 2014;184(3):631-43. https://doi.org/10.1016/j.ajpath.2013.11.019.

25. Shin NR, Whon TW, Bae JW. Proteobacteria: microbial signature of dysbiosis in gut microbiota. Trends Biotechnol. 2015;33(9):496-503. https://doi.org/1 0.1016/j.tibtech.2015.06.011.

26. Ley RE, Turnbaugh PJ, Klein S, Gordon Jl. Microbial ecology: human gut microbes associated with obesity. Nature. 2006;444(7122):1022-3. https:// doi.org/10.1038/4441022a.

27. Yang T, Santisteban MM, Rodriguez V, Li E, Ahmari N, Carvajal JM, et al. Gut dysbiosis is linked to hypertension. Hypertension. 2015:65(6):1331-40. https://doi.org/10.1161/HYPERTENSIONAHA.115.05315.

28. Toral M, Romero M, Rodríguez-Nogales A, Jiménez R, Robles-Vera I, Algieri $F$, et al. Lactobacillus fermentum improves tacrolimus-induced hypertension by restoring vascular redox state and improving eNOS coupling. Mol Nutr Food Res. 2018;62(14):1800033. https://doi.org/10.1 002/mnfr.201800033.

29. Dei-Cas I, Giliberto F, Luce L, Dopazo H, Penas-Steinhardt A. Metagenomic analysis of gut microbiota in non-treated plaque psoriasis patients stratified by disease severity: development of a new psoriasis-microbiome index. Sci Rep. 2020;10(1):12754. https://doi.org/10.1038/s41598-020-69537-3.

30. Janowitz C, Nakamura YK, Metea C, Gligor A, Yu W, Karstens L, et al. Disruption of intestinal homeostasis and intestinal microbiota during experimental autoimmune uveitis. Invest Ophthalmol Vis Sci. 2019;60(1): 420-9. https://doi.org/10.1167/iovs.18-24813.

31. Andriessen EM, Wilson AM, Mawambo G, Dejda A, Miloudi K, Sennlaub F, et al. Gut microbiota influences pathological angiogenesis in obesity-driven choroidal neovascularization. EMBO Mol Med. 2016;8(12):1366-79. https:// doi.org/10.15252/emmm.201606531.

32. Vaiserman A, Romanenko M, Piven L, Moseiko V, Lushchak O, Kryzhanovska $\mathrm{N}$, et al. Differences in the gut Firmicutes to Bacteroidetes ratio across age groups in healthy Ukrainian population. BMC Microbiol. 2020;20(1):221. https://doi.org/10.1186/s12866-020-01903-7.

33. Litvak Y, Byndloss MX, Tsolis RM, Bäumler AJ. Dysbiotic Proteobacteria expansion: a microbial signature of epithelial dysfunction. Curr Opin Microbiol. 2017:39:1-6. https://doi.org/10.1016/j.mib.2017.07.003.

34. Maharshak N, Packey CD, Ellermann M, Manick S, Siddle JP, Huh EY, et al. Altered enteric microbiota ecology in interleukin 10-deficient mice during development and progression of intestinal inflammation. Gut Microbes. 2013;4(4):316-24. https://doi.org/10.4161/gmic.25486.

35. Powell N, Walker AW, Stolarczyk E, Canavan JB, Gökmen MR, Marks E, et al. The transcription factor T-bet regulates intestinal inflammation mediated by 
interleukin-7 receptor+ innate lymphoid cells. Immunity. 2012;37(4):674-84 https://doi.org/10.1016/j.immuni.2012.09.008.

36. Guo M, Wang H, Xu S, Zhuang Y, An J, Su C, et al. Alteration in gut microbiota is associated with dysregulation of cytokines and glucocorticoid therapy in systemic lupus erythematosus. Gut Microbes. 2020;11(6):1758-73. https://doi.org/10.1080/19490976.2020.1768644.

37. Everard A, Lazarevic V, Derrien M, Girard M, Muccioli GG, Neyrinck AM, et al. Responses of gut microbiota and glucose and lipid metabolism to prebiotics in genetic obese and diet-induced leptin-resistant mice. Diabetes. 2011;60(11):2775-86. https://doi.org/10.2337/db11-0227.

38. Larsen N, Vogensen FK, van den Berg FW, Nielsen DS, Andreasen AS, Pedersen BK, et al. Gut microbiota in human adults with type 2 diabetes differs from non-diabetic adults. PLoS One. 2010;5(2):e9085. https://doi.org/1 0.1371/journal.pone.0009085

39. Ye Z, Zhang N, Wu C, Zhang X, Wang Q, Huang $X$, et al. A metagenomic study of the gut microbiome in Behcet's disease. Microbiome. 2018;6(1):135 https://doi.org/10.1186/s40168-018-0520-6.

40. van der Mee-Marquet NL, Bénéjat L, Diene SM, Lemaignen A, Gaïa N, Smet A, et al. A potential new human pathogen belonging to helicobacter genus, Identified in a Bloodstream Infection. Front Microbiol. 2017;8:2533.

41. Nunes-Costa D, Magalhães JD, GF M, Cardoso SM, Empadinhas N. Microbial BMAA and the Pathway for Parkinson's Disease Neurodegeneration. Front Aging Neurosci. 2020;12:26.

42. Bárcena C, Valdés-Mas R, Mayoral P, Garabaya C, Durand S, Rodríguez F, et al. Healthspan and lifespan extension by fecal microbiota transplantation into progeroid mice. Nat Med. 2019;25(8):1234-42. https://doi.org/10.1038/ s41591-019-0504-5.

43. Wu J, Ren W, Li L, Luo M, Xu K, Shen J, et al. Effect of aging and glucagonlike peptide 2 on intestinal microbiota in SD rats. Aging Dis. 2018;9(4):56677. https://doi.org/10.14336/AD.2017.1001.

44. Fielding RA, Reeves AR, Jasuja R, Liu C, Barrett BB, Lustgarten MS. Muscle strength is increased in mice that are colonized with microbiota from highfunctioning older adults. Exp Gerontol. 2019;127:110722. https://doi.org/10.1 016/j.exger.2019.110722.

45. Ding X, Xu Y, Zhang X, Zhang L, Duan G, Song C, et al. Gut microbiota changes in patients with autism spectrum disorders. J Psychiatr Res. 2020; 129:149-59. https://doi.org/10.1016/j.jpsychires.2020.06.032.

46. Bressa C, Bailén-Andrino M, Pérez-Santiago J, González-Soltero R, Pérez M, Montalvo-Lominchar MG, et al. Differences in gut microbiota profile between women with active lifestyle and sedentary women. PLoS One. 2017;12(2):e0171352. https://doi.org/10.1371/journal.pone.01 71352.

47. Baldini F, Hertel J, Sandt E, Thinnes CC, Neuberger-Castillo L, Pavelka L, et al. Parkinson's disease-associated alterations of the gut microbiome predict disease-relevant changes in metabolic functions. BMC Biol. 2020;18(1):62. https://doi.org/10.1186/s12915-020-00775-7.

48. Morotomi M, Nagai F, Sakon H, Tanaka R. Paraprevotella clara gen. Nov., sp. nov. and Paraprevotella xylaniphila sp. nov., members of the family 'Prevotellaceae' isolated from human faeces. Int I Syst Evol Microbiol. 2009, 59(8):1895-900. https://doi.org/10.1099/ijs.0.008169-0.

49. Wang W, Wang J, Li J, Yan P, Jin Y, Zhang R, et al. Cholecystectomy damages aging-associated intestinal microbiota construction. Front Microbiol. 2018:9:1402. https://doi.org/10.3389/fmicb.2018.01402.

50. Hor YY, Lew LC, Jaafar MH, Lau AS, Ong JS, Kato T, et al. Lactobacillus sp improved microbiota and metabolite profiles of aging rats. Pharmacol Res. 2019;146:104312

51. Langille MG, Meehan CJ, Koenig JE, Dhanani AS, Rose RA, Howlett SE, et al. Microbial shifts in the aging mouse gut. Microbiome. 2014;2(1):50. https:// doi.org/10.1186/s40168-014-0050-9.

52. Wexler HM. Bacteroides: the good, the bad, and the nitty-gritty. Clin Microbiol Rev. 2007;20(4):593-621. https://doi.org/10.1128/CMR.00008-07.

53. Sullivan DA, Rocha EM, Aragona P, Clayton JA, Ding J, Golebiowski B, et al. TFOS DEWS II sex, gender, and hormones report. Ocul Surf. 2017;15(3):284333. https://doi.org/10.1016/j.jtos.2017.04.001

54. de Paiva CS. Effects of aging in dry eye. Int Ophthalmol Clin. 2017;57(2):4764. https://doi.org/10.1097/IO.0000000000000170.

55. de Souza RG, de Paiva CS, Alves MR. Age-related autoimmune changes in lacrimal glands. Immune Netw. 2019;19(1):e3. https://doi.org/10.4110/in.20 9.19.e3.

56. Yoon $\mathrm{CH}$, Ryu JS, Hwang HS, Kim MK. Comparative analysis of age-related changes in lacrimal glands and Meibomian glands of a C57BL/6 male mouse model. Int J Mol Sci. 2020;21(11):4169. https://doi.org/10.3390/ ijms21114169.

57. Kappel S, Hawkins P, Mendl MT. To Group or Not to Group? Good Practice for Housing Male Laboratory Mice. Animals (Basel). 2017;7(12):88. https://doi. org/10.3390/ani7120088.

58. Wu ML, Yang XQ, Xue L, Duan W, Du JR. Age-related cognitive decline is associated with microbiota-gut-brain axis disorders and neuroinflammation in mice. Behav Brain Res. 2021;402:113125.

59. Liu A, Lv H, Wang H, Yang H, Li Y, Qian J. Aging increases the severity of colitis and the related changes to the gut barrier and gut microbiota in humans and mice. J Gerontol A Biol Sci Med Sci. 2020;75(7):1284-92. https://doi.org/10.1093/gerona/glz263.

60. Jian C, Luukkonen P, Yki-Järvinen H, Salonen A, Korpela K. Quantitative PCR provides a simple and accessible method for quantitative microbiota profiling. PLoS One. 2020;15(1):e0227285. https://doi.org/10.1371/journal. pone. 0227285

61. Rocha EM, Alves M, Rios JD, Dartt DA. The aging lacrimal gland: changes in structure and function. Ocul Surf. 2008;6(4):162-74. https://doi.org/10.1016/ S1542-0124(12)70177-5.

62. Graber TG, Kim JH, Grange RW, McLoon LK, Thompson LV. C57BL/6 life span study: age-related declines in muscle power production and contractile velocity. Age (Dordr). 2015;37:9773.

63. Lemp MA. Report of the National eye Institute/industry workshop on clinical trials in dry eyes. CLAO J. 1995;21(4):221-32.

64. Kojima T, Nagata T, Kudo H, Muller-Lierheim WGK, van Setten GB, Dogru M, et al. The effects of high molecular weight hyaluronic acid eye drop application in environmental dry eye stress model mice. Int J Mol Sci. 2020; 21(10):3516. https://doi.org/10.3390/ijms21103516.

65. Segata N, Izard J, Waldron L, Gevers D, Miropolsky L, Garrett WS, et al. Metagenomic biomarker discovery and explanation. Genome Biol. 2011; 12(6):R60. https://doi.org/10.1186/gb-2011-12-6-r60.

\section{Publisher's Note}

Springer Nature remains neutral with regard to jurisdictional claims in published maps and institutional affiliations.

Ready to submit your research? Choose BMC and benefit from

- fast, convenient online submission

- thorough peer review by experienced researchers in your field

- rapid publication on acceptance

- support for research data, including large and complex data types

- gold Open Access which fosters wider collaboration and increased citations

- maximum visibility for your research: over $100 \mathrm{M}$ website views per year

At $\mathrm{BMC}$, research is always in progress.

Learn more biomedcentral.com/submissions 